\title{
LIGO as a probe of dark sectors
}

\author{
Fei Huang $\odot,{ }^{1,2, *}$ Veronica Sanz $\odot,{ }^{3,4, \dagger}$ Jing Shu $\odot,{ }^{1,5,6,7,8,9, *}$ and Xiao Xue ${ }^{1,5, \S}$ \\ ${ }^{1}$ CAS Key Laboratory of Theoretical Physics, Institute of Theoretical Physics, Chinese Academy of \\ Sciences, Beijing 100190, China \\ ${ }^{2}$ Department of Physics and Astronomy, University of California, Irvine, California 92697, USA \\ ${ }^{3}$ Instituto de Física Corpuscular (IFIC), Universidad de Valencia-CSIC, E-46980 Valencia, Spain \\ ${ }^{4}$ Department of Physics and Astronomy, University of Sussex, Brighton BN1 9QH, United Kingdom \\ ${ }^{5}$ School of Physical Sciences, University of Chinese Academy of Sciences, Beijing 100049, China \\ ${ }^{6}$ CAS Center for Excellence in Particle Physics, Beijing 100049, China \\ ${ }^{7}$ Center for High Energy Physics, Peking University, Beijing 100871, China \\ ${ }^{8}$ School of Fundamental Physics and Mathematical Sciences, Hangzhou Institute for Advanced Study, \\ UCAS, Hangzhou 310024, China \\ ${ }^{9}$ International Centre for Theoretical Physics Asia-Pacific, Beijing/Hangzhou, China
}

(Received 5 March 2021; revised 9 June 2021; accepted 30 September 2021; published 2 November 2021)

\begin{abstract}
We show how current LIGO data is able to probe interesting theories beyond the Standard Model, particularly dark sectors where a dark Higgs boson triggers symmetry breaking via a first-order phase transition. We use publicly available LIGO O2 data to illustrate how these sectors, even if disconnected from the Standard Model, can be probed by gravitational wave detectors. We link the LIGO measurements with the model content and mass scale of the dark sector, finding that current $\mathrm{O} 2$ data are testing a broad set of scenarios that can be mapped into many different types of dark-sector models where the breaking of $S U(N)$ theories with $N_{f}$ fermions is triggered by a dark Higgs boson at scales $\Lambda \simeq 10^{8}-10^{9} \mathrm{GeV}$ with reasonable parameters for the scalar potential.
\end{abstract}

DOI: 10.1103/PhysRevD.104.095001

\section{INTRODUCTION}

Much of the Universe is dark, and many theories have been built trying to explain it. Our hopes for probing these theories often rely on their possible connection to the standard model (SM) via some form of nongravitational interaction. For example, direct searches for dark matter hinges on some sort of coupling to nucleons or electrons, and constraints on those couplings usually assume a mechanism of communication between the dark sector (DS) and the rest of the Universe, which establishes some form of tracking between these two sectors.

The first observation of gravitational waves (GW) by the LIGO and Virgo Collaborations [1-3] in 2015 initiated a new way to see the Universe. Since then, exciting new observations have provided information about astrophysical

\footnotetext{
*huangf4@uci.edu

†veronica.sanz@uv.es

\#jshu@itp.ac.cn

§xuexiao@itp.ac.cn
}

Published by the American Physical Society under the terms of the Creative Commons Attribution 4.0 International license. Further distribution of this work must maintain attribution to the author(s) and the published article's title, journal citation, and DOI. Funded by SCOAP. objects like black holes [4,5]. However, LIGO is not circumscribed to the detection of mergers [6-9]. The detection, or the lack of a stochastic GW background (SGWB) allows us to explore interesting, nonstandard sectors. We will explain how, with the current public data from LIGO, one can probe plausible DS scenarios, regardless of their nongravitational interaction with visible matter.

The DS could resemble Standard Model dynamics, with new forces, dark Higgs bosons, and states charged under them. Influenced by thermal contributions from the degrees of freedom in the DS, the dark Higgs boson could then experience first-order phase transitions. Many studies have been devoted to the prospects of future interferometers in exploring DS e.g., Ref. [10]. In this paper, we explore the possibilities that LIGO and its current public dataset present and bridge the gap between generic studies of thermal parameters, e.g., Ref. [11], and specific particle-physics models.

The paper is structured as follows. In Sec. II, we first describe the analysis of GWs from first-order phase transitions, then discuss in Sec. III, the connection between the phase-transition thermal parameters with classes of particle-physics models, especially of $S U(N) / S U(N-1)$ DS. We finally link these models to current exclusions set by LIGO in Sec. IV, and in Sec. V, we conclude the discussion. 


\section{GRAVITATIONAL WAVES FROM PHASE TRANSITIONS}

The SGWB is often considered as an isotropic, unpolarized, stationary, and Gaussian background generated by a large number of unresolved GW sources [6-9]. Its power spectrum is characterized by the dimensionless quantity,

$$
\Omega_{\mathrm{GW}}(f) \equiv \frac{1}{\rho_{\text {crit }}} \frac{d \rho_{\mathrm{GW}}}{d \ln f},
$$

where $\rho_{\mathrm{GW}}$ is the energy density of the SGWB, $f$ is the frequency of the GW, and $\rho_{\text {crit }} \equiv 3 c^{2} H_{0}^{2} /(8 \pi G)$ is the critical energy density of the Universe today with $c$ being the speed of light, $H_{0}$ the present-day Hubble constant, and $G$ the gravitational constant.

In general, the total SGWB is a superposition of contributions from all possible astrophysical and cosmological sources. In the absence of a detection, the nondetection of a SGWB sets up an upper limit on the sum of all sources. However, for the SGWB from a particular source, one can always obtain a conservative upper limit on it by assuming that the SGWB is produced predominantly by this source. Therefore, in what follows, we shall assume that the only source of SGWB is an early-Universe phase transition when deriving the upper limit.

The SGWB generated from phase transitions consists of three parts [12-17],

$$
\Omega_{\mathrm{GW}}=\Omega_{\mathrm{col}}+\Omega_{\mathrm{sW}}+\Omega_{\mathrm{turb}},
$$

which correspond to contributions from bubble collisions, sound waves in the fluid, and turbulence, respectively. For models in which gauge bosons acquire masses during the phase transition [18], the contribution from sound waves is typically dominant. For simplicity, we shall assume that the contribution from sound waves is always dominant and neglect the contribution from bubble collisions and turbulence. Nevertheless, the analysis we present can be easily generalized to cases in which other types of contribution become more important. ${ }^{1}$

The phase transition is in general characterized by just a few parameters: the velocity of the bubble wall $v_{w}$, the ratio of the free energy density difference (between the true and false vacuum) and the total energy density $\alpha$, the speed of the phase transition $\beta / H$, and the nucleation temperature $T_{N}$. The explicit definitions of $\alpha$ and $\beta / H$ are given at the beginning of Sec III. With these parameters, the GW power spectrum can be parametrized as ${ }^{2}$

\footnotetext{
${ }^{1}$ In strongly supercooled phase transitions [19-22] or scenarios in which new heavy particles can provide sufficiently large friction [23], contribution from turbulence or bubble collision could also be important.

${ }^{2}$ The standard formula from Ref. [24] is multiplied by a suppression factor due to the finite lifetime of sound waves [2528], whose explicit form is taken from Ref. [28]. It is also shown recently that theoretical uncertainties could change the GW spectrum by orders of magnitude [29].
}

$$
\begin{aligned}
\Omega_{\mathrm{GW}} h^{2} \simeq & 8.5 \times 10^{-6}\left(\frac{g_{*}\left(T_{N}\right)}{100}\right)^{-1 / 3} \Gamma^{2} \bar{U}_{f}^{4}\left(\frac{\beta}{H}\right)^{-1} v_{w} \\
& \times S_{\mathrm{sw}}(f) \Upsilon\left(\tau_{\mathrm{sw}}\right),
\end{aligned}
$$

where $g_{*}\left(T_{N}\right)$ is the effective number of relativistic degrees of freedom, $\Gamma \sim 4 / 3$ is the adiabatic index, $\bar{U}_{f}^{2} \sim(3 / 4) \kappa_{f} \alpha$ is the root-mean-square fluid velocity with the efficiency parameter given by the approximate expressions [15],

$$
\kappa_{f} \approx \begin{cases}\frac{\alpha}{0.73+0.83 \sqrt{\alpha}+\alpha} & v_{w} \rightarrow 1 \\ \frac{\alpha^{2 / 5}}{0.017+(0.997+\alpha)^{2 / 5}} & v_{w} \approx 0.5\end{cases}
$$

the spectral shape,

$$
S_{\mathrm{sw}}(f)=\left(\frac{f}{f_{\mathrm{pk}}}\right)^{3}\left(\frac{7}{4+3\left(f / f_{\mathrm{pk}}\right)^{2}}\right)^{7 / 2},
$$

with the peak frequency,

$f_{\mathrm{pk}} \simeq 8.9 \times 10^{-8} \mathrm{~Hz}\left(\frac{1}{v_{w}}\right)\left(\frac{\beta}{H}\right)\left(\frac{T_{N}}{\mathrm{GeV}}\right)\left(\frac{g_{*}\left(T_{N}\right)}{100}\right)^{1 / 6}$,

and the suppression factor $\Upsilon=1-\left(1+2 \tau_{\mathrm{sw}} H_{*}\right)^{-1 / 2}$ with the lifetime of sound waves $\tau_{\mathrm{sw}} \approx(8 \pi)^{1 / 3} v_{w} /\left(\beta \bar{U}_{f}\right)$.

The shape of $S_{\mathrm{sw}}$ is shown in Fig. 1, noting that $S_{\mathrm{sw}}$ is equal to $\Omega_{\mathrm{GW}}(f) / \Omega_{\mathrm{GW}}\left(f_{\mathrm{pk}}\right)$. In this figure, one observes that varying the peak frequency $f_{\mathrm{sw}}$ amounts to simply shifting the spectrum horizontally. Also note that the asymptotic behavior $-S_{\mathrm{sw}} \sim f^{3}$ for $f \ll f_{\mathrm{sw}}$, whereas $S_{\mathrm{sw}} \sim f^{-4}$ for $f \gg f_{\mathrm{sw}}$.

\section{A. Data analysis}

With this spectrum, we follow the procedure laid out in Refs. [30,31] to compute the upper limit of $\Omega_{\mathrm{GW}}$ for different values of $f_{\mathrm{pk}}$ using data downloaded from the LIGO O2 data release [32] with a $16 \mathrm{kHz}$ sampling frequency. ${ }^{3}$ The data release contains strain data of LIGO detectors at Hanford and Livingston from November 2016 to August 2017. Each downloaded data file covers a $4096 \mathrm{~s}$ period of measurement, and the file name contains the start time of the measurement, which is referred to as the "GPS start time." In each file, there are in general two types of data-the strain time series $h\left(t_{i}\right)$ and some auxiliary data, such as the data quality (DQ) mask label associated with each strain measurement. The DQ mask is a 7-bit binary number, each of which indicates whether a certain type of check is passed (value $=1$ ) or not (value $=0)$. We convert this binary number into a decimal digit such that if

${ }^{3} \mathrm{~A}$ good reference for the analysis pipeline can be found in Ref. [33]. 
$\mathrm{DQ}\left(t_{i}\right)=(0000000)_{10}=0$, there is no data at time $t_{i}$. On the contrary, data are present if this value is nonzero.

The raw strain data are first down sampled to $4 \mathrm{kHz}$ and divided into 50\%-overlapping $192 \mathrm{~s}$ segments. We then select out the time stamps at which both detectors are taking data properly, i.e., the times $t_{i}$ at which both $\mathrm{DQ}_{H}\left(t_{i}\right) \neq 0$ and $\mathrm{DQ}_{L}\left(t_{i}\right) \neq 0$. We then combine continuous time stamps within a file and across neighboring files into larger segments. The segments, whose duration $\geq 600 \mathrm{~s}$, are further picked out to perform a stationarity cut following Refs. [30,34]. When performing the stationarity cut (and, later, when calculating the upper limit), we also notch out frequencies at which the data exhibit narrow band coherent lines that are known to be instrumental or environmental artifacts [35-38]. The list of notched frequencies can be found at Ref. [39].

The selected data are then analyzed using the crosscorrelation method. The cross-correlation statistic for the two detectors is defined as

$$
\hat{C}(f) \equiv \frac{2}{T} \frac{\operatorname{Re}\left[\tilde{s}_{1}^{*}(f) \tilde{s}_{2}(f)\right]}{\gamma_{T}(f) S_{0}(f)},
$$

in which $S_{0}(f)=3 H_{0}^{2} /\left(10 \pi^{2} f^{3}\right), \tilde{s}_{1,2}(f)$ are the Fourier transform of the Hann-windowed strain data of each detector, $T=192 \mathrm{~s}$ is the duration of the data segment used in the Fourier transform, and $\gamma_{T}$ is the overlap reduction function [40]. In the limit where the GW signal is negligible comparing to the instrumental noise, the variance of $\hat{C}(f), \sigma^{2}(f)$, is given by

$$
\sigma^{2}(f)=\frac{1}{2 T \Delta f} \frac{P_{1}(f) P_{2}(f)}{\gamma_{T}^{2}(f) S_{0}^{2}(f)},
$$

where $\Delta f=1 / 32 \mathrm{~Hz}$, and $P_{1,2}(f)$ are the one-sided noise power spectra of each detector. For each $192 \mathrm{~s}$ segment, we define the broadband estimator $\hat{\Omega}_{\text {ref }}$ of $\Omega_{\mathrm{GW}}$,

$$
\hat{\Omega}_{\mathrm{ref}} \equiv \frac{\sum_{k} w^{-1}\left(f_{k}\right) \hat{C}\left(f_{k}\right) \sigma^{-2}\left(f_{k}\right)}{\sum_{k} w^{-2}\left(f_{k}\right) \sigma^{-2}\left(f_{k}\right)},
$$

where $f_{k}$ are discrete frequencies in the band $20-1726 \mathrm{~Hz}$ with the interval of $1 / 32 \mathrm{~Hz},{ }^{4}$ and the weight function is optimized by choosing $w(f)=\Omega_{\mathrm{GW}}\left(f_{\text {ref }}\right) / \Omega_{\mathrm{GW}}(f)$ where $f_{\text {ref }}$ is a reference frequency. This choice ensures the normalization $\left\langle\hat{\Omega}_{\text {ref }}\right\rangle=\Omega_{\mathrm{GW}}\left(f_{\text {ref }}\right)$. Note that, the optimal weight function is inversely proportional to $S_{\mathrm{sw}}$, and, since $f_{k} \in[20-1726] \mathrm{Hz}$, the spectral shape within this band can transform from a descending power law, to one with a peak in between, and eventually, to an ascending power law

\footnotetext{
${ }^{4}$ The lower limit is due to the loss of sensitivity at lower frequencies caused by detector noises $[41,42]$, whereas the upper limit is chosen to prevent aliasing effects from down sampling the raw data [31].
}

when $f_{\mathrm{pk}}$ increases (see Fig. 1). The variance of the optimal estimator is given by

$$
\sigma_{\Omega}^{-2}=\sum_{k} w^{-2}\left(f_{k}\right) \sigma^{-2}\left(f_{k}\right)
$$

We calculate $\hat{\Omega}_{\text {ref }}$ and $\sigma_{\Omega}^{2}$ for all the $192 \mathrm{~s}$ segments, and the ensemble average over all the segments is obtained from

$$
\begin{gathered}
\left\langle\sigma_{\Omega}\right\rangle=\frac{1}{\sqrt{\sum_{I} \sigma_{\Omega, I}^{-2}}}, \\
\left\langle\hat{\Omega}_{\mathrm{ref}}\right\rangle=\frac{\sum_{I} \hat{\Omega}_{\mathrm{ref}, I} \sigma_{\Omega, I}^{-2}}{\sum_{I} \sigma_{\Omega, I}^{-2}} .
\end{gathered}
$$

In the absence of any detection signal, we set the $95 \%$ confidence level upper limit by

$$
\Omega_{\mathrm{GW}}^{\text {up } \lim }\left(f_{\text {ref }}\right)=2 \times\left\langle\sigma_{\Omega}\right\rangle\left(f_{\text {ref }}\right) .
$$

Apparently, the upper limit also depends on the choice of $f_{\text {ref }}$ - a inserting the optimal weights $w(f)$ in Eq. (10), we have $\sigma_{\Omega} \propto \Omega_{\mathrm{GW}}\left(f_{\text {ref }}\right)$ and thus, $\Omega_{\mathrm{GW}}^{\text {up } \lim }\left(f_{\text {ref }}\right) \propto \Omega_{\mathrm{GW}}\left(f_{\text {ref }}\right)$ according to Eq. (13). As a result, the upper limit at one reference frequency can be easily related to that at another reference frequency,

$$
\Omega_{\mathrm{GW}}^{\mathrm{up} \lim }\left(f_{\text {ref }}^{\prime}\right)=\Omega_{\mathrm{GW}}^{\mathrm{up}} \lim \left(f_{\mathrm{ref}}\right) \frac{\Omega_{\mathrm{GW}}\left(f_{\mathrm{ref}}^{\prime}\right)}{\Omega_{\mathrm{GW}}\left(f_{\mathrm{ref}}\right)} .
$$

In Fig. 2, we show the upper limit at $f_{\text {ref }}=25 \mathrm{~Hz}$, which lies in the frequency band that LIGO is most sensitive

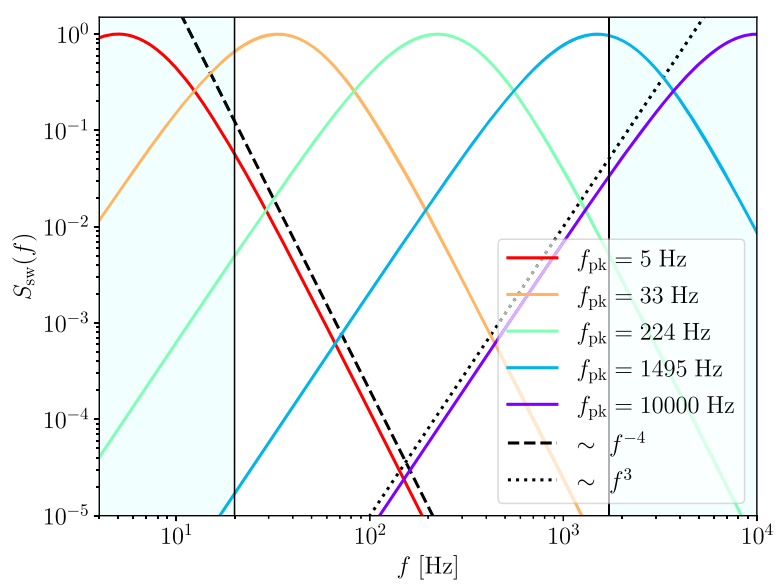

FIG. 1. The spectral shape of $S_{\mathrm{sw}}(f)$ by varying the peak frequency. The dashed and dotted black lines indicate the asymptotic behavior. The two solid vertical lines at 20 and $1726 \mathrm{~Hz}$ show the minimum and maximum frequency considered when deriving the constraints - only the part of the spectrum between the two boundaries are taken into account in our data analysis. 


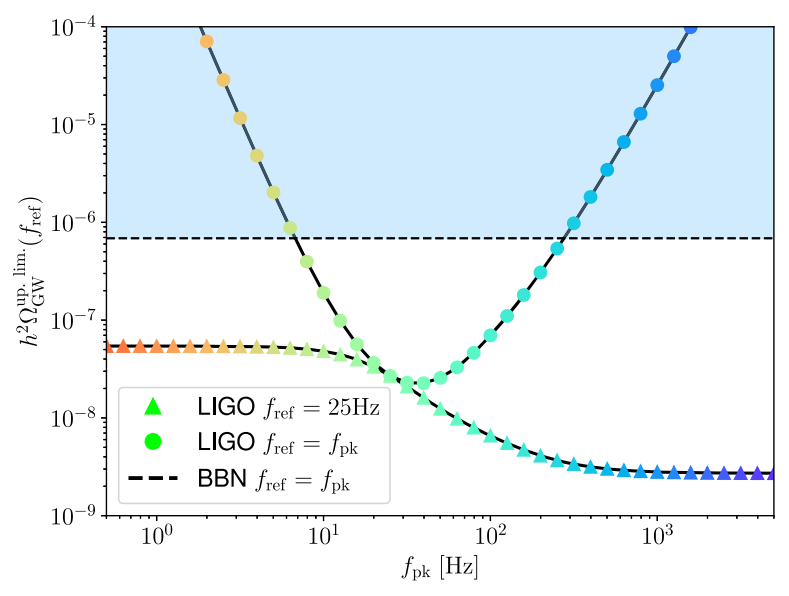

FIG. 2. The $95 \%$ confidence level upper limit of $\Omega_{\mathrm{GW}}\left(f_{\text {ref }}\right)$ for different peak frequencies $f_{\mathrm{pk}}$. The triangles and circles correspond to the upper limit at $25 \mathrm{~Hz}$ and at $f_{\mathrm{pk}}$, respectively. Note that each $f_{\mathrm{pk}}$ represents a specific $\Omega_{\mathrm{GW}}$ considered in the analysis (see Fig. 1), and the upper limit depends on the choice of $f_{\text {ref }}$. The relation between upper limits at different $f_{\text {ref }}$ is given by Eq. (14). The dashed horizontal line corresponds to the BBN bound at $f_{\mathrm{pk}}$.

to $[30,31,43]$. The triangles correspond to the data points that we actually calculated, and the curve is an interpolation of them. Notice that, for $f_{\mathrm{pk}} \lesssim 10 \mathrm{~Hz}$ or $f_{\mathrm{pk}} \gtrsim 10^{3} \mathrm{~Hz}$, the bound approaches a constant, and there is a smooth transition for intermediate $f_{\mathrm{pk}}$. This is expected from the continuous transition from the ascending and descending power laws within the 20-1726 Hz band (again, see Fig. 1). Obviously, LIGO provides a stronger constraint on ascending spectra than on the descending spectra, and the difference can be as large as an order of magnitude.

In order to make connection with the thermal parameters in Eq. (3), we use Eq. (14) to map the constraint at $f_{\text {ref }}=25 \mathrm{~Hz}$ to that at $f_{\mathrm{pk}}$. The curve made by circles in Fig. 2 shows the result of this mapping. Note that, for $f_{\mathrm{pk}}$ very small or very large, the upper bound from LIGO becomes substantially weaker than the constraint from the big bang nucleosynthesis (BBN), which is obtained by requiring $h^{2} \int d \ln f \Omega_{\mathrm{GW}}(f)<1 \times 10^{-6} \quad[9,44]$. with $h$ being the dimensionless Hubble parameter.

In what follows, we shall discuss how to obtain the thermal parameters from models of phase transitions, and how the LIGO constraint can be utilized to constrain those models.

\section{SCENARIOS FOR PHASE TRANSITIONS AND THEIR THERMAL PARAMETERS}

Typically, one represents phase transitions as driven by the dynamics of a scalar field that transitions from one vacuum to another under the influence of the evolving thermal potential. In that context, the thermal parameters $\beta / H$ and $\alpha$ are defined by

$$
\begin{gathered}
\frac{\beta}{H}=\left.T \frac{d\left(S_{E} / T\right)}{d T}\right|_{T=T_{N}}, \\
\alpha=\left.\frac{1}{\rho_{N}}\left(\Delta V-T \Delta \frac{d V}{d T}\right)\right|_{T=T_{N}},
\end{gathered}
$$

where $S_{E}$ is the Euclidean action, $V$ is the thermal potential of the scalar field, $\rho_{N}=g_{\star}\left(T_{N}\right) \pi^{2} T_{N}^{4} / 30$, and the bubble nucleation temperature can be obtained by solving [45]

$$
\frac{S_{E}}{T_{N}} \approx 177-4 \ln \left(\frac{T_{N}}{\mathrm{GeV}}\right)-2 \ln g_{\star}\left(T_{N}\right) .
$$

On the other hand, the calculation of the bubble-wall velocity $v_{w}$ for a particular model is highly nontrivial. Therefore, instead of directly calculating it, we follow the customary convention of considering a few reference values, $v_{w}=0.5$ and 1 .

To connect the thermal parameters $\alpha$ and $\beta / H$ to specific models, we consider two classes of potentials consisting of competing terms with alternating signs,

$$
\begin{aligned}
& V(H, T)=\frac{1}{2} m^{2}(T) h_{D}^{2}-c_{3}(T) h_{D}^{3}+\frac{1}{4} \lambda(T) h_{D}^{4} \\
& V(H, T)=\frac{1}{2} m^{2}(T) h_{D}^{2}-\frac{1}{4} \lambda(T) h_{D}^{4}+c_{6}(T) h_{D}^{6},
\end{aligned}
$$

where the coefficients of the scalar field $h_{D}$ are all positive at the time of transition. It has been pointed out that most dark phase transitions can be mapped onto these effective scenarios [10], e.g., in a DS where its particles acquire masses from a dark Higgs boson as its gauge group $S U(N)$ breaks into $S U(N-1)$, the potentials in Eqs. (18) and (19) can be realized with renormalizable operators and nonrenormalizable sextet interaction, respectively. ${ }^{5}$

In the following, we shall discuss the dark Higgs boson $-S U(N) / S U(N-1)$ models with renormalizable and nonrenormalizable operators, specifically using the parametrization from Ref. [10].

\section{A. Models with renormalizable operators}

For the type of potential in Eq. (18), we can parametrize zero-temperature parameters as

$$
m^{2}(0)=-\frac{\Lambda^{4}}{v_{D}^{2}}, \quad \lambda(0)=\frac{\Lambda^{4}}{v_{D}^{4}},
$$

in which $v_{D}$ is the zero temperature vacuum expectation value, and $\Lambda$ is the scale of the potential. With this parametrization, the finite temperature potential can be expressed as

\footnotetext{
${ }^{5} \mathrm{We}$ assume the temperature is the same in both the dark and SM sector, which is the case if the DS is in kinetic equilibrium with the SM sector. Otherwise, one needs to consider the evolution of the DS temperature [46,47].
} 


$$
\begin{aligned}
V(H, T)= & \Lambda^{4}\left[-\frac{1}{2}\left(\frac{h_{D}}{v_{D}}\right)^{2}+\frac{1}{4}\left(\frac{h_{D}}{v_{D}}\right)^{4}\right] \\
& +\frac{T^{4}}{2 \pi^{2}}\left[\sum_{i \in \text { bosons }} n_{i} J_{B}\left(\frac{m_{i}^{2}}{T^{2}}\right)-\sum_{i \in \text { fermions }} n_{i} J_{F}\left(\frac{m_{i}^{2}}{T^{2}}\right)\right] \\
= & \Lambda^{4}\left\{\left[-\frac{1}{2}+\left(\frac{1}{8}+\frac{N_{G}}{24}\right) \frac{T^{2}}{v_{D}^{2}}\right.\right. \\
& \left.+\frac{3}{24} N_{\mathrm{GB}} \frac{g^{2}}{4} \frac{T^{2} v_{D}^{2}}{\Lambda^{4}}+y^{2} N_{f} \frac{T^{2}}{48} \frac{v_{D}^{2}}{\Lambda^{4}}\right]\left(\frac{h_{D}}{v_{D}}\right)^{2} \\
& \left.-\left[N_{\mathrm{GB}}\left(\frac{g^{2}}{4}\right)^{3 / 2} \frac{1}{4 \pi} \frac{v_{D}^{3} T}{\Lambda^{4}}\right]\left(\frac{h_{D}}{v_{D}}\right)^{3}+\frac{1}{4}\left(\frac{h_{D}}{v_{D}}\right)^{4}\right\}
\end{aligned}
$$

where $N_{\mathrm{GB}}=2 N-1$ is the number of gauge bosons that couple to the dark Higgs boson with coupling constant $g$ and get a mass from the dark Higgs interactions, $N_{G}=$ $2 N-1$ is the number of Goldstone bosons, and $N_{f}=$ $N \times N_{\mathrm{FL}}$ is the number of self-adjoint fermions with Yukawa coupling $y$, where $N_{\mathrm{FL}}$ is the number of flavors. Note that for Dirac fermions, one would need to double the number of degrees of freedom. For simplicity, we assume the Yukawa coupling is universal for those fermions.

In the second equality, the following high temperature expansions are used. Moreover, the following high temperature expansions are used:

$J_{B}\left(\frac{m^{2}}{T^{2}}\right) \sim \frac{m^{2}}{24 T^{2}}-\frac{m^{3}}{12 \pi T^{3}}, \quad J_{F}\left(\frac{m^{2}}{T^{2}}\right) \sim-\frac{m^{2}}{48 T^{2}}$,

in which the field dependent masses can be read as

$$
\begin{gathered}
m_{H}^{2}=\partial_{h_{D}}^{2} V=\Lambda^{4}\left(3 \frac{h_{D}^{2}}{v^{4}}-\frac{1}{v^{2}}\right), \\
m_{G}^{2}=\frac{1}{h_{D}} \partial_{h_{D}} V=\Lambda^{4}\left(\frac{h_{D}^{2}}{v_{D}^{4}}-\frac{1}{v_{D}^{2}}\right), \\
m_{\mathrm{GB}}=\frac{g h_{D}}{2} \\
m_{f}=\frac{y h_{D}}{\sqrt{2}} .
\end{gathered}
$$

Note that in the second line of Eq. (21), only the massive gauge bosons are taken into account; i.e., the higher order terms $\left(\sim m^{3} / T^{3}\right)$ from Goldstone bosons and the dark Higgs bosons are neglected. Besides, the part of the expansion which gives rise to terms independent of $h_{D}$ is also neglected, since it only amounts to a constant shift in the potential $V$. With all these ingredients, the mapping from Eq. (21) to the temperature-dependent parameters in Eq. (18) is straightforward by matching the terms with the same powers of $h_{D}$.
Equation (21) is also often written in the following form:

$$
\begin{aligned}
V(H, T)= & \Lambda^{4}(T)\left[\left(\frac{3-4 \eta(T)}{2}\right)\left(\frac{h_{D}}{v_{D}(T)}\right)^{2}\right. \\
& \left.-\left(\frac{h_{D}}{v_{D}(T)}\right)^{3}+\eta(T)\left(\frac{h_{D}}{v_{D}(T)}\right)^{4}\right],
\end{aligned}
$$

in which the minimum of the potential can be easily obtained by minimizing the potential,

$$
v_{D}(T)=\frac{3 c_{3}(T)+\sqrt{9 c_{3}^{2}(T)-4 m^{2}(T) \lambda^{2}(T)}}{2 \lambda(T)} .
$$

By identifying

$$
c_{3}(T)=\frac{\Lambda^{4}(T)}{v_{D}^{3}(T)}, \quad \frac{\lambda(T)}{4}=\eta(T) \frac{\Lambda^{4}(T)}{v_{D}^{4}(T)},
$$

one finds

$\eta(T)=\frac{\lambda(T) v_{D}(T)}{4 c_{3}(T)}, \quad \Lambda(T)=\left(c_{3}(T) v_{D}^{3}(T)\right)^{1 / 4}$.

With these, for $\eta(T) \in[0.51,0.65]$, the effective action can be fitted by [45]

$$
\frac{S_{E}}{T}=\frac{v_{D}^{3}(T)}{T \Lambda^{2}(T)} 10^{a+b \frac{\ln (T)-\left.0.75\right|^{c}}{\ln (T)-\left.0.5\right|^{d}}}
$$

in which the fitting parameters $a=-71.06, b=71.62$, $c=0.008805$, and $d=0.009263$.

\section{B. Models with nonrenormalizable operators}

Similar to the previous case, we parametrize the zerotemperature parameters in Eq. (19) as

$$
m^{2}(0)=(2-3 \eta) \frac{\Lambda^{4}}{v_{D}^{2}}, \quad \lambda(0)=4 \frac{\Lambda^{4}}{v_{D}^{4}}, \quad c_{6}(0)=\eta \frac{\Lambda^{4}}{v_{D}^{6}} .
$$

The finite temperature potential then becomes

$$
\begin{aligned}
V(H, T)= & \Lambda^{4}\left[(2-3 \eta)\left(\frac{h_{D}}{v_{D}}\right)^{2}-\left(\frac{h_{D}}{v_{D}}\right)^{4}+\eta\left(\frac{h_{D}}{v_{D}}\right)^{6}\right] \\
& +\frac{T^{4}}{2 \pi^{2}}\left[\sum_{i \in \text { bosons }} n_{i} J_{B}\left(\frac{m_{i}^{2}}{T^{2}}\right)-\sum_{i \in \text { fermions }} n_{i} J_{F}\left(\frac{m_{i}^{2}}{T^{2}}\right)\right] \\
= & \Lambda^{4}\left\{\left[2-3 \eta-\left(\frac{1}{2}+\frac{N_{G}}{6}\right) \frac{T^{2}}{v_{D}^{2}}\right.\right. \\
& \left.+\frac{3}{24} N_{\mathrm{GB}} \frac{g^{2}}{4} \frac{T^{2} v_{D}^{2}}{\Lambda^{4}}+y^{2} N_{f} \frac{T^{2}}{48} \frac{v_{D}^{2}}{\Lambda^{4}}\right]\left(\frac{h_{D}}{v_{D}}\right)^{2} \\
& \left.-\left[1-\frac{\left(30+6 N_{G}\right) \eta T^{2}}{24 v_{D}^{2}}\right]\left(\frac{h_{D}}{v_{D}}\right)^{4}+\eta\left(\frac{h_{D}}{v_{D}}\right)^{6}\right\},
\end{aligned}
$$


with which the mapping to the finite-temperature parameters in Eq. (19) is straightforward. Note that, in the hightemperature expansion, the cubic term is assumed to be subdominant; i.e., we have only kept the part proportional to $m^{2} / T^{2}$ [10]. Moreover, the field-dependent masses of the Goldstone bosons and the dark Higgs bosons, which go into the thermal corrections are

$$
\begin{aligned}
& m_{H}^{2}=\Lambda^{4}\left[\frac{2(2-3 \eta)}{v_{D}^{2}}-\frac{12 h_{D}^{2}}{v_{D}^{4}}+\frac{30 \eta h_{D}^{4}}{v_{D}^{6}}\right], \\
& m_{G}^{2}=\Lambda^{4}\left[\frac{2(2-3 \eta)}{v_{D}^{2}}-\frac{4 h_{D}^{2}}{v_{D}^{4}}+\frac{6 \eta h_{D}^{4}}{v_{D}^{6}}\right] .
\end{aligned}
$$

The terms proportional to $\eta h_{D}^{4} / v_{D}^{4}$ are what give rise to the thermal correction of the quartic term.

Just as in the previous case, we can also write Eq. (33) in terms of temperature-dependent parameters,

$$
\begin{aligned}
V(H, T)= & \Lambda^{4}(T)\left[(2-3 \eta(T))\left(\frac{h}{v_{D}(T)}\right)^{2}-\left(\frac{h}{v_{D}(T)}\right)^{4}\right. \\
& \left.+\eta(T)\left(\frac{h}{v_{D}(T)}\right)^{6}\right]
\end{aligned}
$$

in which

$v_{D}(T)=\left(\frac{\lambda(T)+\sqrt{\Lambda^{2}(T)-24 c_{6}(T) m^{2}(T)}}{12 c_{6}(T)}\right)^{1 / 2}$

is obtained by minimizing the potential. Obviously, $\eta(T)$ can be obtained by solving

$$
\lambda(T)=4 \Lambda(T)^{4} / v(T)^{4}, \quad c_{6}(T)=\eta(T) \Lambda(T)^{4} / v_{D}(T)^{6},
$$

which gives

$$
\eta(T)=4 c_{6}(T) v_{D}^{2}(T) / \lambda(T) .
$$

Following Ref. [10], the Euclidean action in this case can be fitted by

$$
S_{E}=\frac{v_{D}^{3}(T)}{\Lambda^{2}(T)} 10^{\sum_{i=1}^{3} a_{i}(\eta(T)-2 / 3)^{i}}
$$

for $\eta(T) \in[0.51,0.65]$ with $a_{i}=(-17.446,-132.404$, $-763.744)$.

Finally, at temperatures relevant for the dark phase transition, we shall assume that particles in the DS are all relativistic and are the only degrees of freedom in addition to the SM. Therefore, the effective number of relativistic degrees of freedom will be given by

$$
\begin{aligned}
g_{\star}\left(T_{N}\right) \approx & 106.75+3 N_{\mathrm{GB}}+2\left(N^{2}-1-N_{\mathrm{GB}}\right) \\
& +1+7 / 8 \times 2 N_{f},
\end{aligned}
$$

where we have included all degrees of freedom from the $\mathrm{SM}$ as well as all gauge bosons and dark fermions charged under $S U(N)$.

After specifying the two classes of models, we can then compute the thermal parameters for each of them. For all of the cases, we shall take the rank of the gauge group $N=2,5$, the number of flavors $N_{\mathrm{FL}}=3$, the Yukawa coupling $y=1$, and the scale of the potential $\Lambda \in\left[10^{6}, 10^{10}\right] \mathrm{GeV}$. For models with renormalizable couplings, we scan over the parameter space where the ratio $v_{D} / \Lambda \in[0.5,4]$ and the coupling $g \in[0.1,1]$, whereas for models with nonrenormalizable couplings, we fix $g=1$ and scan over $v_{D} / \Lambda \in[1,5]$ and $\eta \in[0.5,2]$.

We first look for the critical temperature for the firstorder phase transition $T_{c}$, at which degenerate minima appear with a potential barrier between them and then search down for the nucleation temperature $T_{N}$ by solving Eq. (17) using Eq. (31) or Eq. (40). Parameters which cannot give rise to a barrier between the degenerate minima, or which lead to values of $\eta\left(T_{N}\right)$ outside of fitting range of Eq. (31) or Eq. (40), are discarded. Once $T_{N}$ is found, $\beta / H$ and $\alpha$ can then be easily calculated.

In the following section, we then compare the obtained thermal parameters against the LIGO constraints that we derived in Sec. II in order to test whether LIGO has the ability to probe the phase-transition models.

\section{EXPLORING DARK SECTORS WITH LIGO}

In Fig. 3, we present the LIGO constraints on the thermal parameters at $95 \%$ confidence level along with the BBN constraint. In the left panels, the constraints are evaluated with $v_{w}=0.5$, whereas the bounds in the right panels correspond to $v_{w}=1$.

With $v_{w}$ fixed, the thermal parameters $\left(\beta / H, T_{N}, \alpha\right)$ constitute a three-dimensional space. We find that the excluded region can be conveniently projected on the $\beta / H-T_{N}$ plane as different contours of $\alpha$ do not intersect. Within one contour, any value of $\alpha$ larger than that of the contour is excluded. This motivates us to use color variation to indicate $\alpha$. Therefore, regions in the 2D plots where the LIGO ellipsoidal contours have a color lighter than the BBN vertical contours suggest that LIGO sets a better bound than BBN, and vice versa.

We also plot the thermal parameters obtained from different models defined by $\Lambda, N$, and $N_{\mathrm{FL}}$ in Fig. 3 . We show examples with $N=2$ and 5 , and $N_{\mathrm{FL}}=3$, to show the behavior expected from the choices on matter content and gauge groups. Moreover, we fix $g=1$ and $y=1$, though similar behavior is found for similar $\mathcal{O}(1)$ values. 

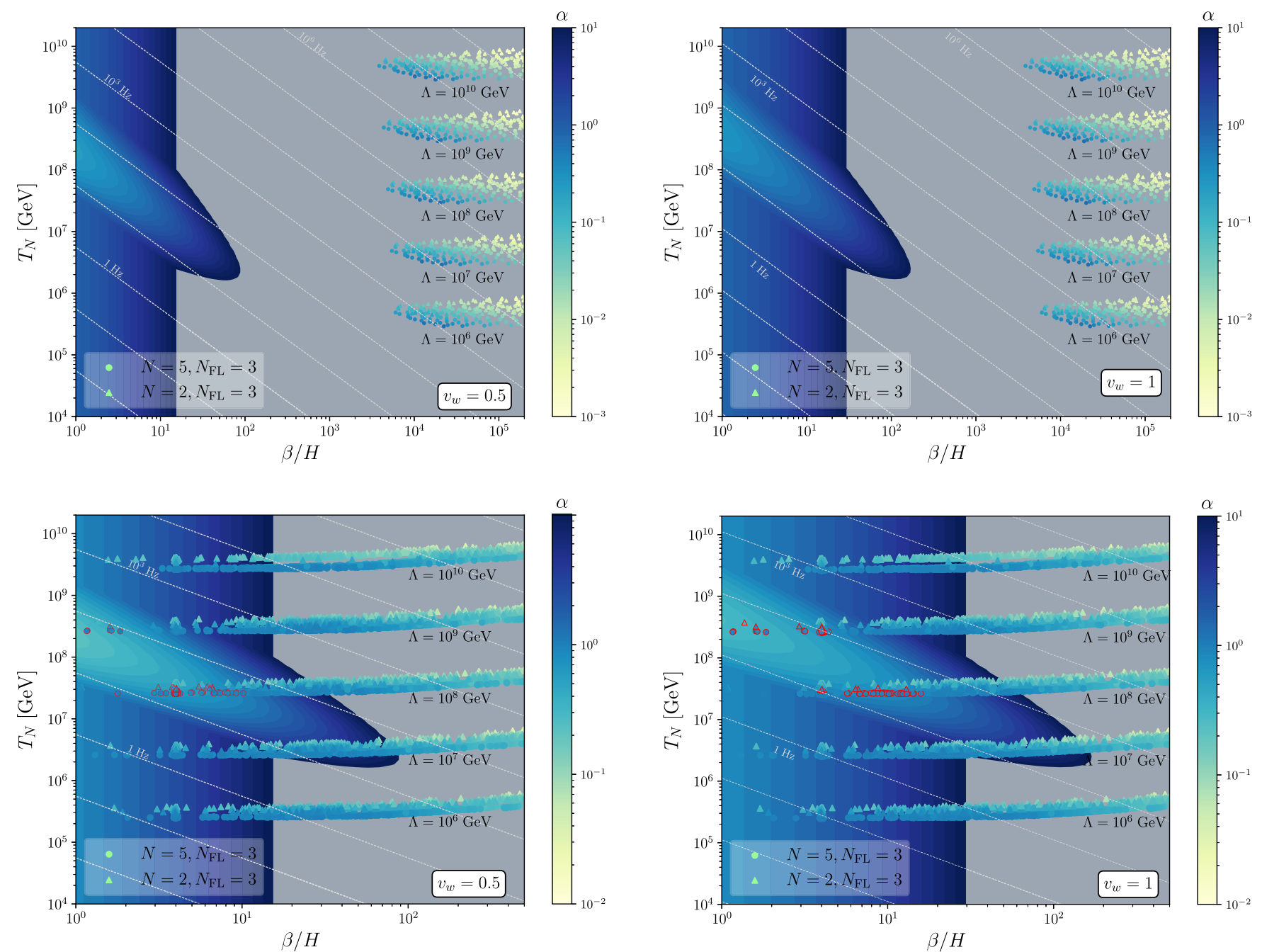

FIG. 3. The 95\% confidence level exclusion region from LIGO (curved contours) and the constraint from BBN (vertical contours) projected on the $\beta / H-T_{N}$ plane. The values of $\alpha$ are represented by the color variation, and $f_{\mathrm{pk}}$ is indicated by the white dashed contours. The markers in the top and bottom panels correspond to models with renormalizable and nonrenormalizable operators, respectively. If any marker contained within a contour has a larger $\alpha$ value than the corresponding contour then the corresponding combination of thermal parameters is excluded. Such excluded markers are also circled in red. Note that the range of the color bar is different in the top and bottom panels.

If a set of thermal parameters obtained from our models lies within a contour and has a larger $\alpha$ (darker color) than the corresponding contour, then this set of thermal parameter is excluded at $95 \%$ CL To guide the eye, markers with red borders represent the thermal parameters that are excluded by the LIGO but allowed by BBN. We restrict that $\beta / H \geq 1$ since smaller values of $\beta / H$ indicates that the average size of the bubbles is larger than the horizon size upon collision, which is unphyiscal. For relatively slow transitions $(\beta / H \lesssim 10)$, a detailed analysis may be necessary to ensure that the bubbles of the true vacuum percolate $[48,49]$. However, this is beyond the scope of this paper. In addition, we also restrict $\alpha \leq 1$ to ensure a radiation-dominated Universe during the phase transition.
From the top panels, we find that the constraint from LIGO is still unable to probe the renormalizable classes of models, as markers representing the thermal parameters cannot reach the excluded region. However, in the bottom panels, we indeed see how LIGO is able to probe the nonrenormalizable classes. In particular, we notice that the scales $\Lambda \sim 10^{8}-10^{9} \mathrm{GeV}$ are better constrained, and models with larger $N$ and $N_{\mathrm{FL}}$ are more constrained as they tend to produce a larger $\alpha$. The range of zerotemperature parameters where LIGO currently has sensitivity is given by

$\frac{m}{\Lambda} \in[0.14,0.35], \quad \lambda \in[0.3,1.1], \quad c_{6} \Lambda^{2} \in[0.01,0.1]$, 
which are reasonable choices of parameter space and indicate that LIGO is testing interesting DS theories.

\section{CONCLUSIONS}

When discussing DS and their GW signatures, we usually consider future probes like LISA. Here, we have shown that LIGO is already probing interesting DS scenarios.

For first-order phase transitions and the LIGO data, the emphasis has been placed on performing effective analyses; e.g., bounds from LIGO $\mathrm{O} 3$ has been explored recently using parametrizations of the power spectrum [11]. In this paper, we take a complementary step and focus on examining whether reasonable particle-physics models could be related to the tested regions.

We have focused on two classes of models which cover a broad set of DS scenarios and capable of producing GW signatures. Those benchmark models have been identified as more promising for strong first-order phase transition from DS and allow a simple parametrization in terms of the group rank $N$ and the number of flavors $N_{\mathrm{FL}}$ [10]. We find that scales around $10^{8}-10^{9} \mathrm{GeV}$ are better probed by LIGO $\mathrm{O} 2$. We also find that the sensitive regions correspond to moderate values of $N$ and $N_{\mathrm{FL}}$, evidencing that LIGO is not testing extreme regions in the parameter space. Of course, various other types of models for phase transitions could also generate $\mathrm{GW}$ with frequencies relevant for LIGO, e.g., models motivated by grand unification theories $[50,51]$ and models for confinement-deconfinement phase transitions [52,53].

Our results motivate a more systematic study of the particle-physics scenarios that LIGO is able to test. We emphasize again that traditional direct or indirect searches for dark particles assume that the DS interacts nongravitationally with the SM. However, since gravity is universal, methods for probing the DS via gravitational effects, such as structure formation (see Refs. [54,55] and references therein for recent progress) and gravitational waves, do not rely on those assumptions. These gravitational effects therefore offer unique opportunities to access DS even in the absence of nongravitational interactions.

\section{ACKNOWLEDGMENTS}

We would like to thank Djuna Croon for conversations at the beginning of this project. V. S. acknowledges support from the United Kingdom Science and Technology Facilities Council ST/L000504/1. J. S. and F. H. are supported by the National Natural Science Foundation of China under Grants No. 12025507, No. 11690022, and No. 11947302; and is supported by the Strategic Priority Research Program and Key Research Program of Frontier Science of the Chinese Academy of Sciences under Grants No. XDB21010200, No. XDB23010000, and No. ZDBSLY-7003. F. H. is also supported by the National Science Foundation of China under Grants No. 12022514 and No. 11875003.
[1] B. P. Abbott et al. (LIGO Scientific and Virgo Collaborations), Phys. Rev. Lett. 116, 061102 (2016).

[2] J. Aasi, B. Abbott, R. Abbott, T. Abbott, M. Abernathy, K. Ackley, C. Adams, T. Adams, P. Addesso, R. Adhikari et al., Classical Quantum Gravity 32, 074001 (2015).

[3] F. a. Acernese, M. Agathos, K. Agatsuma, D. Aisa, N. Allemandou, A. Allocca, J. Amarni, P. Astone, G. Balestri, G. Ballardin et al., Classical Quantum Gravity 32, 024001 (2014).

[4] B. Abbott, R. Abbott, T. Abbott, S. Abraham, F. Acernese, K. Ackley, C. Adams, R. Adhikari, V. Adya, C. Affeldt et al., Phys. Rev. X 9, 031040 (2019).

[5] R. Abbott, T. Abbott, S. Abraham, F. Acernese, K. Ackley, A. Adams, C. Adams, R. Adhikari, V. Adya, C. Affeldt et al., Phys. Rev. X 11, 021053 (2021).

[6] B. Allen and J. D. Romano, Phys. Rev. D 59, 102001 (1999).

[7] M. Maggiore, Phys. Rep. 331, 283 (2000).

[8] T. Regimbau, Res. Astron. Astrophys. 11, 369 (2011).

[9] C. Caprini and D. G. Figueroa, Classical Quantum Gravity 35, 163001 (2018).
[10] D. Croon, V. Sanz, and G. White, J. High Energy Phys. 08 (2018) 203.

[11] A. Romero, K. Martinovic, T. A. Callister, H.-K. Guo, M. Martínez, M. Sakellariadou, F.-W. Yang, and Y. Zhao, Phys. Rev. Lett. 126, 151301 (2021).

[12] C. Caprini, R. Durrer, and G. Servant, Phys. Rev. D 77, 124015 (2008).

[13] S. J. Huber and T. Konstandin, J. Cosmol. Astropart. Phys. 09 (2008) 022.

[14] C. Caprini, R. Durrer, and G. Servant, J. Cosmol. Astropart. Phys. 12 (2009) 024.

[15] J. R. Espinosa, T. Konstandin, J. M. No, and G. Servant, J. Cosmol. Astropart. Phys. 06 (2010) 028.

[16] M. Hindmarsh, S. J. Huber, K. Rummukainen, and D. J. Weir, Phys. Rev. Lett. 112, 041301 (2014).

[17] M. Hindmarsh, Phys. Rev. Lett. 120, 071301 (2018).

[18] D. Bodeker and G. D. Moore, J. Cosmol. Astropart. Phys. 05 (2017) 025.

[19] J. Ellis, M. Lewicki, J. M. No, and V. Vaskonen, J. Cosmol. Astropart. Phys. 06 (2019) 024.

[20] J. Ellis, M. Lewicki, and V. Vaskonen, J. Cosmol. Astropart. Phys. 11 (2020) 020. 
[21] M. Lewicki and V. Vaskonen, Eur. Phys. J. C 81, 437 (2021).

[22] M. Lewicki and V. Vaskonen, Eur. Phys. J. C 80, 1003 (2020).

[23] A. Azatov and M. Vanvlasselaer, J. Cosmol. Astropart. Phys. 01 (2021) 058.

[24] D. J. Weir, Phil. Trans. R. Soc. A 376, 20170126 (2018).

[25] M. Hindmarsh, S. J. Huber, K. Rummukainen, and D. J. Weir, Phys. Rev. D 96, 103520 (2017); 101, 089902(E) (2020).

[26] J. Ellis, M. Lewicki, and J. M. No, J. Cosmol. Astropart. Phys. 04 (2019) 003.

[27] C. Caprini et al., J. Cosmol. Astropart. Phys. 03 (2020) 024.

[28] H.-K. Guo, K. Sinha, D. Vagie, and G. White, J. Cosmol. Astropart. Phys. 01 (2021) 001.

[29] D. Croon, O. Gould, P. Schicho, T. V. I. Tenkanen, and G. White, J. High Energy Phys. 04 (2021) 055.

[30] B. P. Abbott et al. (LIGO Scientific and Virgo Collaborations), Phys. Rev. Lett. 118, 121101 (2017); 119, 029901(E) (2017).

[31] B. Abbott et al. (LIGO Scientific and Virgo Collaborations), Phys. Rev. D 100, 061101 (2019).

[32] https://www.gw-openscience.org/data/.

[33] S. Kandhasamy, Searches for stochastic gravitational waves and long gravitational wave transients in LIGO S5 data, Ph.D. thesis, Minnesota University, 2013.

[34] B. P. Abbott et al. (LIGO Scientific and VIRGO Collaborations), Nature (London) 460, 990 (2009).

[35] B. P. Abbott, R. Abbott, T. Abbott, M. Abernathy, F. Acernese, K. Ackley, M. Adamo, C. Adams, T. Adams, P. Addesso et al., Classical Quantum Gravity 33, 134001 (2016).

[36] E. Thrane, N. Christensen, and R. M. Schofield, Phys. Rev. D 87, 123009 (2013).

[37] E. Thrane, N. Christensen, R. M. Schofield, and A. Effler, Phys. Rev. D 90, 023013 (2014).
[38] P. Covas, A. Effler, E. Goetz, P. Meyers, A. Neunzert, M. Oliver, B. Pearlstone, V. Roma, R. Schofield, V. Adya et al., Phys. Rev. D 97, 082002 (2018).

[39] https://dcc.ligo.org/LIGO-T1900058/public.

[40] N. Christensen, Phys. Rev. D 46, 5250 (1992).

[41] B. P. Abbott et al., Phys. Rev. D 93, 112004 (2016); 97, 059901(A) (2018).

[42] A. Buikema et al. (aLIGO Collaboration), Phys. Rev. D 102, 062003 (2020).

[43] R. Abbott et al. (LIGO Scientific, Virgo, and KAGRA Collaborations), Phys. Rev. D 104, 022004 (2021).

[44] E. H. Tanin and T. Tenkanen, J. Cosmol. Astropart. Phys. 01 (2021) 053.

[45] D. Croon and G. White, J. High Energy Phys. 05 (2018) 210.

[46] M. Breitbach, J. Kopp, E. Madge, T. Opferkuch, and P. Schwaller, J. Cosmol. Astropart. Phys. 07 (2019) 007.

[47] M. Fairbairn, E. Hardy, and A. Wickens, J. High Energy Phys. 07 (2019) 044.

[48] A. H. Guth and E. J. Weinberg, Nucl. Phys. B212, 321 (1983).

[49] M. S. Turner, E. J. Weinberg, and L. M. Widrow, Phys. Rev. D 46, 2384 (1992).

[50] D. Croon, T. E. Gonzalo, and G. White, J. High Energy Phys. 02 (2019) 083.

[51] D. Croon, T. E. Gonzalo, L. Graf, N. Košnik, and G. White, Front. Phys. 7, 76 (2019).

[52] J. Halverson, C. Long, A. Maiti, B. Nelson, and G. Salinas, J. High Energy Phys. 05 (2021) 154.

[53] W.-C. Huang, M. Reichert, F. Sannino, and Z.-W. Wang, Phys. Rev. D 104, 035005 (2021).

[54] K. R. Dienes, F. Huang, J. Kost, S. Su, and B. Thomas, Phys. Rev. D 101, 123511 (2020).

[55] K. R. Dienes, F. Huang, J. Kost, K. Manogue, and B. Thomas, arXiv:2101.10337. 